\title{
Expression of $\alpha$-crystallin in the retina of human sympathetic ophthalmia
}

\author{
SATORU KASE ${ }^{1,2}$, BEERAN B. MEGHPARA ${ }^{3}$, SUSUMU ISHIDA $^{2}$ and NARSING A. RAO ${ }^{1,4}$ \\ ${ }^{1}$ Doheny Eye Institute, Los Angeles, CA, USA; ${ }^{2}$ Hokkaido University Graduate School of Medicine, \\ Sapporo, Japan; ${ }^{3}$ University of Illinois at Chicago, Chicago, IL, USA; ${ }^{4}$ Department of Ophthalmology, \\ Keck School of Medicine, University of Southern California, Los Angeles, CA, USA
}

Received July 20, 2011; Accepted October 11, 2011

DOI: $10.3892 / \mathrm{mmr} .2011 .653$

\begin{abstract}
Sympathetic ophthalmia (SO) is a bilateral, granulomatous, intraocular inflammation that occurs following a penetrating injury to one eye, and has the potential to cause blindness of both eyes. The aim of this study was to examine the expression of $\alpha$-crystallin and to detect apoptotic cells in the retina of human eyes with SO. Five globes, including three with SO and two age-matched normal appearing retinae, were examined. Formalin-fixed, paraffin-embedded tissue sections were submitted to hematoxylin and eosin staining and immunohistochemistry with anti- $\alpha \mathrm{A}$ and $\alpha \mathrm{B}$-crystallin antibodies . Apoptotic cells were detected using the terminal deoxynucleotidyl transferase dUTP nick end labeling (TUNEL) method, and double-staining immunohistochemistry was conducted together with the TUNEL reaction. In normal-appearing retina, $\alpha$ A-crystallin immunoreactivity was predominantly detected in the cytoplasm of photoreceptors, where $\alpha \mathrm{B}$-crystallin was less marked. In SO globes, granulomatous inflammation was noted in the choroid, whereas the retina and choriocapillaris were preserved. Immunoreactivity for $\alpha$ A-crystallin was detected in the retina, as well as in the cytoplasm and inner/ outer photoreceptor segments. By contrast, $\alpha \mathrm{B}$-crystallin was weakly noted in the SO retina. Double-staining immunohistochemistry revealed no TUNEL-positive photoreceptors in the retina displaying high immunoreactivity for $\alpha$ A-crystallin, but photoreceptor apoptosis was noted where expression of $\alpha \mathrm{A}$-crystallin was relatively low. The present study demonstrated that $\alpha \mathrm{A}$-crystallin was up-regulated in the cytoplasm of photoreceptors in the SO retina. This may play a protective role in the suppression of photoreceptor apoptosis associated with intraocular inflammation.
\end{abstract}

Correspondence to: Dr Satoru Kase, Department of Ophthalmology, Laboratory of Ocular Cell Biology and Visual Science, Hokkaido University Graduate School of Medicine, Nishi 7, Kita 15, Kita-ku, Sapporo 060-8638, Japan

E-mail: kaseron@med.hokudai.ac.jp

Key words: $\alpha$-crystallin, apoptosis, retina, sympathetic ophthalmia

\section{Introduction}

Crystallins were primarily identified as major structural proteins of the eye lens. The two $\alpha$-crystallins, $\alpha \mathrm{A}$ and $\alpha \mathrm{B}$, are the principal members of the small heat shock protein (Hsp) family (1). Although the crucial function of $\alpha$-crystallins is to act as molecular chaperones, their expression and chaperone activity vary under varying pathologic conditions (2-4). Mao et al demonstrated the anti-apoptotic function of $\alpha$-crystallins by blocking pro-apoptotic mitochondrial pathways, thereby inhibiting the activation of downstream apoptotic events (3). We recently demonstrated that $\alpha$ A-crystallin was up-regulated in the diabetic retina (5), which is possibly correlated with protection of retinal cells from apoptotic signals during progression of diabetic retinopathy. Moreover, a variety of experimental inflammatory models have revealed that up-regulation of $\alpha \mathrm{A}$ - or $\alpha \mathrm{B}$-crystallin protected specific cells from apoptotic signals associated with inflammation (6-8).

Sympathetic ophthalmia (SO) is a bilateral, granulomatous, intraocular inflammation that occurs following a penetrating injury to one eye, and has the potential to cause blindness of both eyes (9). Although the pathogenesis of SO is not completely understood, it is believed to be caused by an altered tolerance to uveal antigen, which involves a cell-mediated immunity (9). In human eyes with SO, leukocytes initially infiltrate the uvea that is typically absent in the choriocapillaris and retina $(9,10)$. We recently demonstrated that immune-mediated mitochondrial oxidative stress led to photoreceptor apoptosis and subsequent vision loss in SO globes (11).

Experimental autoimmune uveoretinitis (EAU) is a highly reproducible animal model for human endogenous uveitis, including SO. The rodent model of EAU has been used to study T-cell mediated inflammatory processes in the retina (12). Induction of Hsp70 leads to amelioration of EAU through suppressed antigen-specific T-cell proliferation (13). Rao et al demonstrated that photoreceptors selectively up-regulated $\alpha \mathrm{A}$-crystallin to protect themselves against immune-mediated mitochondrial oxidative stress in a murine model of EAU (6). These results suggest that Hsp may play an essential role in the suppression of human endogenous uveitis.

This study was conducted to examine the expression of $\alpha$-crystallins in the retina by immunohistochemistry, and to detect apoptotic cells in human SO globes. 


\section{Materials and methods}

The Institutional Review Board of the University of Southern California (CA, USA) approved our use of human specimens obtained from the file of Doheny Eye Institute, Pathology Laboratory (CA, USA). All procedures conformed to the Declaration of Helsinki for research involving human subjects. Three formalin-fixed, paraffin-embedded human globes with a diagnosis of $\mathrm{SO}$ were retrieved from the archives of A. Ray Irvine Jr, Pathology Laboratory of the Doheny Eye Institute, Los Angeles, CA, USA. All patients had the traumatized eye, commonly referred to as the exciting eye, while the fellow eye was referred to as the sympathizing eye. All globes examined in this study were sympathizing eyes. The age of the patients varied between 38 and 58 years, with a mean age of 46.7. All eyes were removed since the patients had no light perception and the eyes were painful. We also analyzed two histologically normal appearing retinae obtained from age-matched patients with uveal melanoma. Prepared cut sections $(5-\mu \mathrm{m})$ were submitted to hematoxylin and eosin staining as well as immunohistochemistry.

Immunohistochemistry. The slides were dewaxed, rehydrated and rinsed in phosphate-buffered saline (PBS) twice for $10 \mathrm{~min}$. As a pretreatment, microwave-based antigen retrieval was performed in $10 \mathrm{mM}$ citrate buffer (pH 6.0). These slides were incubated with $3 \%$ hydrogen peroxide for $10 \mathrm{~min}$ and then with normal goat serum for $30 \mathrm{~min}$. Sections were incubated with anti- $\alpha$ A-crystallin (1:100; Stressgen, Belgium) and $\alpha B$-crystallin (1:100; Stressgen) rabbit polyclonal antibodies at room temperature for $2 \mathrm{~h}$. Binding of the primary antibody was localized with the FITC-conjugated anti-rabbit secondary antibody (Jackson ImmunoResearch Laboratories, West Grove, PA, USA) for $30 \mathrm{~min}$. Negative controls consisted of FITCconjugated mouse IgG incubated without treatment with the primary antibody. Slides were examined using a Zeiss LSM510 (Zeiss, Thornwood, NY, USA) confocal microscope.

Double-staining immunohistochemistry with terminal deoxynucleotidyl transferase dUTP nick end labeling (TUNEL) reaction. Serial sections $(5-\mu \mathrm{m})$ were cut for TUNEL assay to evaluate distribution of the TUNEL-positive reaction in the same part as the immunoreaction with $\alpha$-crystallins. An in situ Cell Death Detection Fluorescein kit (Roche, Indianapolis, IN, USA) was used for the TUNEL assay. The slides were dewaxed, rehydrated and rinsed twice for $10 \mathrm{~min}$ in PBS. These slides were incubated with $3 \%$ hydrogen peroxide for $10 \mathrm{~min}$, then permeabilized with $20 \mu \mathrm{g} / \mathrm{ml}$ of proteinase $\mathrm{K}$ at room temperature for $10 \mathrm{~min}$. Texas red label with enzyme solution was added to each slide and incubated in a humidified chamber at $37^{\circ} \mathrm{C}$ for $1 \mathrm{~h}$. DNase-pretreated slides were used as positive controls and slides without added enzyme were used as negative controls. Following washing, sections were incubated with anti- $\alpha$ A-crystallin rabbit polyclonal antibodies at room temperature for $2 \mathrm{~h}$. Binding of the primary antibody was localized with the FITC-conjugated anti-rabbit secondary antibody (Jackson ImmunoResearch Laboratories) for $30 \mathrm{~min}$. Apoptotic cells were revealed by confocal microscopy and were defined by the presence of perinuclear chromatin condensation and apoptotic bodies.

\section{Results}

In the age-matched, normal-appearing retina, the ganglion cell layer (GCL), inner nuclear layer (INL) and outer nuclear layer (ONL) were clearly identified (Fig. 1a). $\alpha$ A-crystallin immunoreactivity was predominantly detected in the cytoplasm of photoreceptors (Fig. 1b-d), not in the photoreceptor inner and outer segments (IS/OS) (Fig. 1b). Expression of $\alpha \mathrm{B}$-crystallin was weakly observed in the retina (Fig. 1e and f). The negative control, in which the respective antibody was omitted, displayed no staining in the retina (data not shown). The two crystallins were immunopositive in the cytoplasm of lens epithelial cells in all sections examined in this study, and served as an internal positive control (data not shown).

Immunoreactivity for $\alpha$-crystallins in SO globes. All three SO globes displayed typical histologic features of SO with characteristic diffuse, non-necrotizing and granulomatous inflammation involving the uvea (Fig. 2a). The choroid was markedly thickened and infiltrated by lymphocytes associated with epithelioid and giant cells. Melanin-laden macrophages were intermingled in the choroid. No caseous necrosis was noted. The choriocapillaris was spared from inflammatory cell infiltration (Fig. 2b and c). The inflammation did not extend into the retina, where INL and ONL were clearly present (Fig. $2 b$ and c). Immunoreactivity for $\alpha \mathrm{A}$-crystallin was highly expressed in the retina, including ONL and INL (Fig. 2e and f). Moreover, photoreceptor IS/OS displayed high immunoreactivity for $\alpha$ A-crystallin (Fig. 2e). $\alpha$ A-crystallin immunoreactivity was, however, occasionally weak in the retina. By contrast, $\alpha \mathrm{B}$-crystallin was faintly detected in almost the entire retina and Bruch's membrane (Fig. $2 \mathrm{~h}$ and i).

Correlation with $\alpha A$-crystallin immunoreactivity and TUNEL-positive cells. Parikh et al recently demonstrated that photoreceptor apoptosis occurred on the retina of SO (11). Therefore, double-staining immunoreactivity with TUNEL reaction was conducted to determine whether immunolocalization of $\alpha$ A-crystallin and photoreceptor apoptotic cells are related. No TUNEL-positive cells were detected in the retina (Fig. 3a) where $\alpha$ A-crystallin immunoreactivity was markedly detected (Fig. 3b and c). By contrast, as shown in Fig. 3, TUNEL-positive apoptotic cells were observed in ONL (Fig. $3 \mathrm{~d}$, arrows), where $\alpha \mathrm{A}$-crystallin immunoreactivity was relatively weak in the cytoplasm (Fig. 3e and f, arrows).

\section{Discussion}

This study demonstrated that $\alpha \mathrm{A}$-crystallin is expressed in the cytoplasm of photoreceptors in the normal-appearing retina without inflammation. In human SO globes, granulomatous inflammation was initially present in the choroid, sparing the choriocapillaris and sensory retina. The current study revealed that $\alpha \mathrm{A}$-crystallin, although not $\alpha \mathrm{B}$-crystallin, was selectively expressed in the retina, as well as in the cytoplasm and in the IS/OS of photoreceptors. These results suggest that $\alpha \mathrm{A}$-crystallin expression is up-regulated, and that the immunoreactivity is to be found in the IS/OS of photoreceptors in $\mathrm{SO}$ globes. 


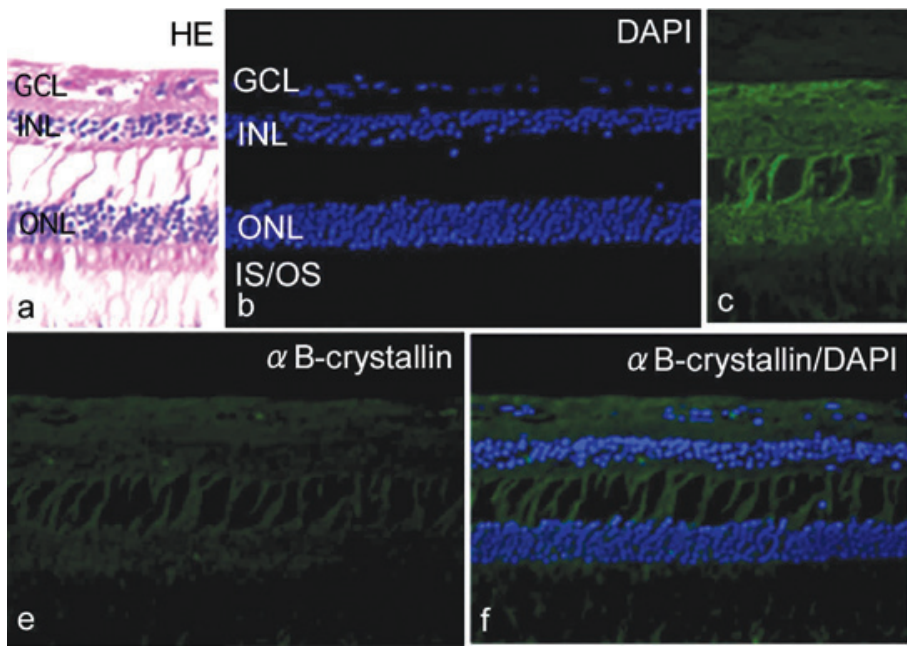

Figure 1. (a) Hematoxylin and eosin staining. (b, d and f; blue) DAPI nuclear staining. (c and d; green) Immunodetection of $\alpha$ A-crystallin and (e and f; green) $\alpha \mathrm{B}$-crystallin in normal appearing retina. (a and b) Three nuclear layers consisting of the ganglion cell layer (GCL), inner nuclear layer (INL), outer nuclear layer (ONL) and photoreceptor inner and outer segment (IS/OS) are clearly confirmed. (c and d) $\alpha$ A-crystallin immunoreactivity is predominantly detected in the cytoplasm of photoreceptors and is weakly noted in GCL and INL. (e and f) Immunoreactivity for $\alpha \mathrm{B}$-crystallin is barely detected. DAPI, 4',6-diamidino-2-phenylindole.
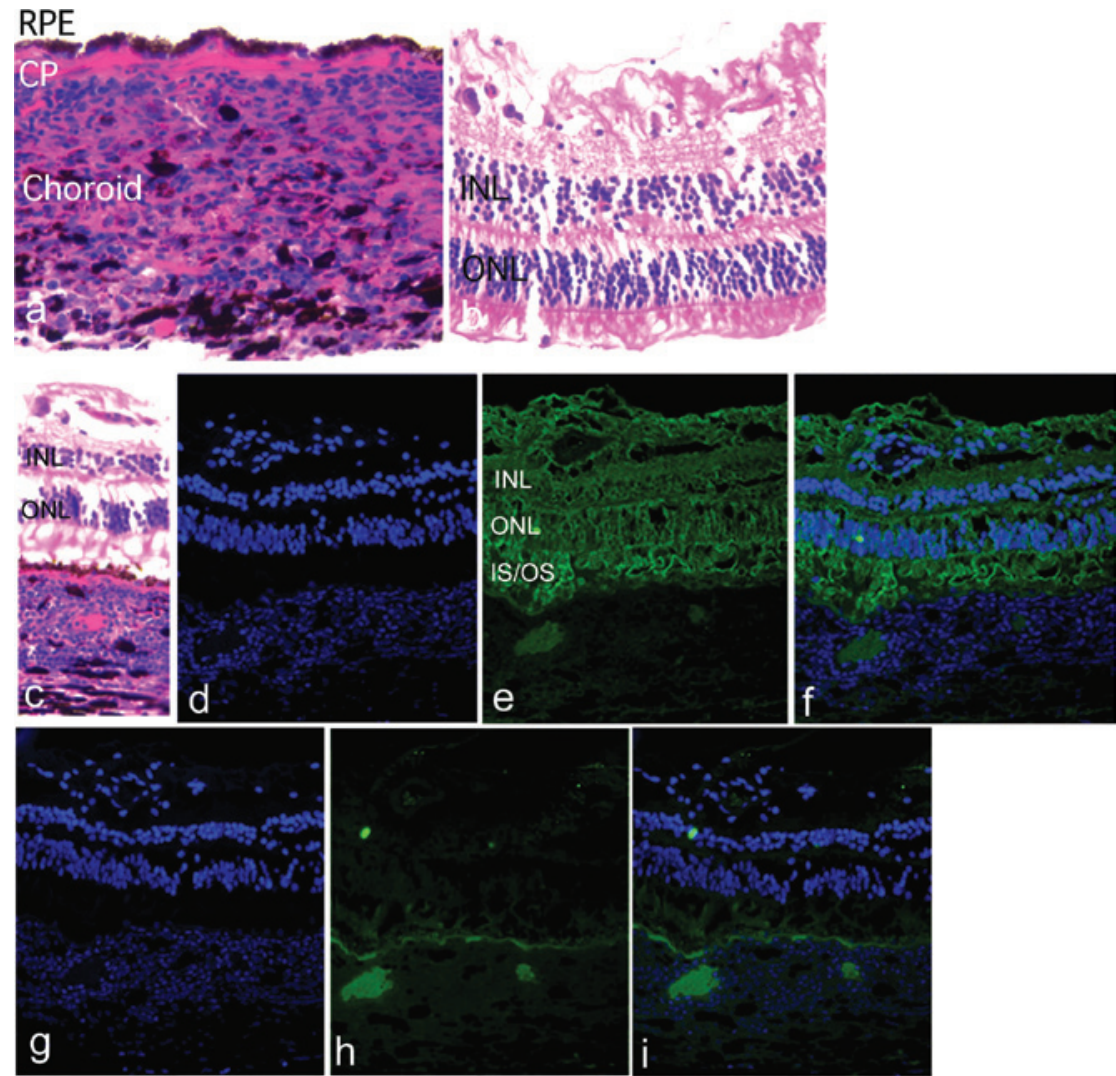

Figure 2. (a-c) Hematoxylin and eosin staining. (d, f, g and i; blue) DAPI nuclear staining. (e and f; green) Expression of $\alpha$ A- and $\alpha \mathrm{B}$ - (h and i; green) crystallin in the retina of sympathetic ophthalmia. (a) Inflammatory cells made up of lymphocytes, epithelioid cells and melanin-laden macrophages infiltrate the choroid. (b and c) Retinal pigment epithelium (RPE), choriocapillaris (CP) and retina are preserved, and the inner nuclear layer (INL) and outer nuclear layer (ONL) are clearly confirmed in the retina. (e and f) Immunoreactivity for $\alpha$ A-crystallin is highly expressed in the retina including photoreceptors and INL. (e) Moreover, photoreceptor inner and outer segments (IS/OS) reveal high immunoreactivity for $\alpha \mathrm{A}$-crystallin. (h and i) By contrast, $\alpha \mathrm{B}$-crystallin is faintly detected in the retina, while Bruch's membrane displays weak expression of $\alpha \mathrm{B}$-crystallin. (e, $\mathrm{f}, \mathrm{h}$ and i) Neither $\alpha \mathrm{A}$ - nor $\alpha \mathrm{B}$-crystallin expression is observed in choroidal inflammation. DAPI, 4',6-diamidino-2-phenylindole.

Apoptotic cell death of photoreceptors occurs in the retina of SO without inflammatory cell infiltration and may cause a loss of vision (11). We clearly demonstrated that photoreceptor apoptosis could be observed in the retina, where $\alpha \mathrm{A}$-crystallin immunoreactivity was relatively low. On the other hand, in the retina where $\alpha$ A-crystallin immunoreactivity was markedly detected, no TUNEL-positive signals were observed. Rao et al found that $\alpha \mathrm{A}$-crystallin was expressed in the photoreceptors 
TUNEL/DAPI

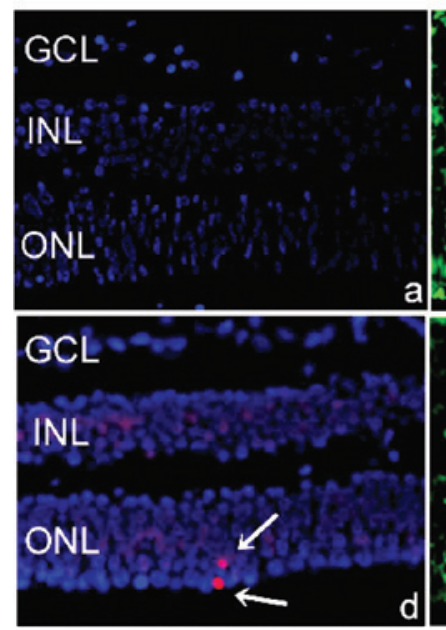

$\alpha$ A-crystallin
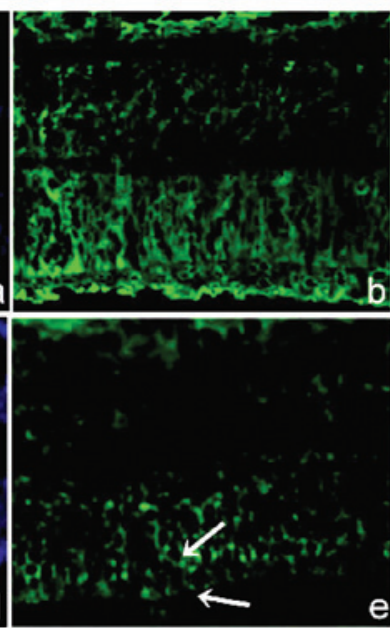
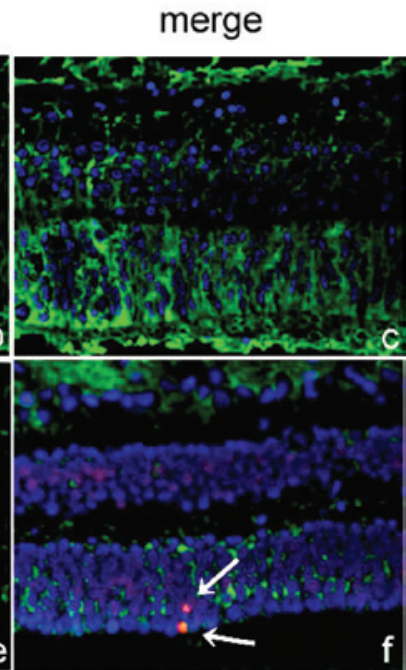

Figure 3. Double staining immunohistochemistry with TUNEL reaction in the retina of sympathetic ophthalmia. (a) TUNEL-positive cells are not detected in this part of the retina including the ganglion cell layer (GCL), inner nuclear layer (INL) and outer nuclear layer (ONL), where (b and c) $\alpha \mathrm{A}$-crystallin immunoreactivity is markedly detected. (b and c) Immunoreactivity for $\alpha$ A-crystallin is also detected in photoreceptor segments. (d, arrows) TUNEL-positive apoptotic cells are observed in ONL, (e and f; arrows) where $\alpha \mathrm{A}$-crystallin immunoreactivity is weakly detected. TUNEL, terminal deoxynucleotidyl transferase dUTP nick end labeling.

and protected them from apoptotic signals in an EAU model (6). In actuality, $\alpha$ A-crystallin exerts an anti-apoptotic function by blocking pro-apoptotic mitochondrial pathways, thereby inhibiting the activation of downstream apoptotic events (3). Therefore, strong expression of $\alpha \mathrm{A}$-crystallin in photoreceptors may have a protective role against apoptotic signals in SO.

Recently, studies have evaluated the pathogenesis of retinal damage in intraocular inflammation using an EAU model. Increased generation of reactive oxygen and nitric oxide species was observed in the photoreceptor mitochondria, indicating oxidative stress, prior to inflammatory cell infiltration in EAU (14). Such oxidative damage in the mitochondria may be the initial event leading to retinal degeneration in EAU (15). Oxidative stress leads to up-regulation of a host of Hsp, including $\alpha$-crystallins (16). Rao et al demonstrated that photoreceptors selectively up-regulated $\alpha \mathrm{A}$-crystallin against immune-mediated mitochondrial oxidative stress by EAU involving the retina (6). We recently demonstrated that mitochondrial oxidative stress occurs in the photoreceptor inner segment in SO globes of humans (11). These results suggest that the $\alpha \mathrm{A}$-crystallin up-regulation observed in this study may result from photoreceptor oxidative stress in SO.

The potential role of $\alpha \mathrm{B}$-crystallin in the pathology of various inflammatory models has been extensively studied (17). $\alpha \mathrm{B}$-crystallin is believed to have a suppressive effect on immunity and an anti-apoptotic role in glia and endothelial cells in experimental inflammatory and angiogenesis models, respectively $(7,18)$. In fact, recombinant $\alpha \mathrm{B}$-crystallin protein reduced the number of inflammatory foci and apoptotic glial cell death in the model (7). Whiston et al identified the critical role of $\alpha \mathrm{B}$-crystallin in protecting the retina during inflammation using a murine model of Staphylococcus aureus-induced endophthalmitis (8). These results suggest $\alpha \mathrm{B}$-crystallin may be a therapeutic target for human inflammatory diseases. In contrast to past publications, this study demonstrated that $\alpha \mathrm{A}$-crystallin, although not $\alpha \mathrm{B}$-crystallin, responded to the inflammation in human SO globes; these findings have been supported by EAU animal models (6). Therefore, the overexpression model of $\alpha \mathrm{A}$-crystallin and application of the recombinant protein into the EAU model should prove the therapeutic role of $\alpha \mathrm{A}$-crystallin in intraocular inflammation.

\section{Acknowledgements}

This study was supported in part by NIH, EY 017347.

\section{References}

1. Andley UP: Crystallins in the eye: function and pathology. Prog Retin Eye Res 26: 78-98, 2007.

2. Andley UP, Song Z, Wawrousek EF, Fleming TP and Bassnett S: Differential protective activity of alpha A- and alphaB-crystallin in lens epithelial cells. J Biol Chem 275: 36823-36831, 2000.

3. Mao YW, Liu JP, Xiang H and Li DW: Human alphaA- and alphaB-crystallins bind to $\mathrm{Bax}$ and $\mathrm{Bcl}-\mathrm{X}(\mathrm{S})$ to sequester their translocation during staurosporine-induced apoptosis. Cell Death Differ 11: 512-526, 2004.

4. Liu JP, Schlosser R, Ma WY, Dong Z, Feng H, Liu L, Huang XQ, Liu Y and Li DW: Human alphaA- and alphaB-crystallins prevent UVA-induced apoptosis through regulation of PKCalpha, RAF/MEK/ERK and AKT signaling pathways. Exp Eye Res 79: 393-403, 2004

5. Kase S, Ishida $S$ and Rao NA: Increased expression of alphaA-crystallin in human diabetic eye. Int J Mol Med: 28: 505-511, 2011.

6. Rao NA, Saraswathy S, Wu GS, Katselis GS, Wawrousek EF and Bhat S: Elevated retina-specific expression of the small heat shock protein, alphaA-crystallin, is associated with photoreceptor protection in experimental uveitis. Invest Ophthalmol Vis Sci 49: 1161-1171, 2008.

7. Ousman SS, Tomooka BH, van Noort JM, Wawrousek EF, O'Conner K, Hafler DA, Sobel RA, Robinson WH and Steinman L: Protective and therapeutic role for alphaB-crystallin in autoimmune demyelination. Nature 448: 474-479, 2007.

8. Whiston EA, Sugi N, Kamradt MC, Sack C, Heimer SR, Engelbert M, Wawrousek EF, Gilmore MS, Ksander BR and Gregory MS: alphaB-crystallin protects retinal tissue during Staphylococcus aureus-induced endophthalmitis. Infect Immun 76: 1781-1790, 2008.

9. Rathinam SR and Rao NA: Sympathetic ophthalmia following postoperative bacterial endophthalmitis: a clinicopathologic study. Am J Ophthalmol 141: 498-507, 2006. 
10. Rao NA, Robin J, Hartmann D, Sweeney JA and Marak GE Jr: The role of the penetrating wound in the development of sympathetic ophthalmia experimental observations. Arch Ophthalmol 101: 102-104, 1983.

11. Parikh JG, Saraswathy S and Rao NA: Photoreceptor oxidative damage in sympathetic ophthalmia. Am J Ophthalmol 146: 866.e2-875.e2, 2008.

12. Agarwal RK and Caspi RR: Rodent models of experimental autoimmune uveitis. Methods Mol Med 102: 395-419, 2004.

13. Kitamei H, Kitaichi N, Yoshida K, et al: Association of heat shock protein 70 induction and the amelioration of experimental autoimmune uveoretinitis in mice. Immunobiology 212: 11-18, 2007.

14. Saraswathy S and Rao NA: Photoreceptor mitochondrial oxidative stress in experimental autoimmune uveitis. Ophthalmic Res 40: 160-164, 2008.
15. Khurana RN, Parikh JG, Saraswathy S, Wu GS and Rao NA: Mitochondrial oxidative DNA damage in experimental autoimmune uveitis. Invest Ophthalmol Vis Sci 49: 3299-3304, 2008.

16. Morimoto RI: Stress, aging, and neurodegenerative disease. N Engl J Med 355: 2254-2255, 2006.

17. Masilamoni JG, Jesudason EP, Bharathi SN and Jayakumar R: The protective effect of alpha-crystallin against acute inflammation in mice. Biochim Biophys Acta 1740: 411-420, 2005.

18. Kase S, He S, Sonoda S, Kitamura M, Spee C, Wawrousek E, Ryan SJ, Kannan R and Hinton DR: alphaB-crystallin regulation of angiogenesis by modulation of VEGF. Blood 115: 3398-3406, 2010 . 